\title{
Die Datenschutzerklärung
}

Liebe Leserinnen, liebe Leser, einem Keimling sieht nicht jedermann an, wozu er sich auswachsen kann. Das mag auch für den Datenschutz gelten. Auch er war einmal klein und am Keimen. Nun ist er fast 40 Jahre als. Man fragt sich:Wie war er, als er klein war? Sieht er sich noch ähnlich?

Er erschien seinerzeit rechtzeitig vor der einsetzenden Datenflut, die er eindämmen sollte. Davor gab es also noch keinen konkreten Leidensdruck, an dem man sich hätte orientieren können. So war der Datenschutz zunächst eine akademische Angelegenheit. Es galt: Die Datenverarbeitung kann eine Waffe in der Hand der Mächtigen sein. Die Kleinen sind zu schützen. Aber wovor und wie? Die Gefahr stand zwar vor der Tür, aber ohne klare Konturen. Es wurde diskutiert. Man fand sich zusammen bei hypothetischen Vorstellungen darüber, wie und wo sich wohl der Datenverarbeitungsteufel des Betroffenen bemächtigen könnte. Und man überlegte, was dagegen zu tun sei. Das hatte ein erstes Bundesdatenschutz- und eine Reihe von Landesdatenschutzgesetzen zur Folge, mit der Erwartung, dass es so kommen würde wie sich vorgestellt.

Richtungsweisend war den Beteiligten die Überzeugung gewesen, dass der Betroffene das Recht haben müsse, mitzubestimmen, welche seiner Daten von der Speichernden Stelle erfasst und verarbeitet werden dürfen. Um das durchsetzen zu können, sollte er Rechte auf Auskunft sowie auf Berichtigung, Löschung und Sperrung seiner Daten haben. Man nahm an, dass er, ein mündiger Bürger, mit diesen Instrumenten für sein Recht selbst sorgen könne. Er musste dazu wissen, wer seine Daten verarbeitet. Wenn er es nicht wissen konnte, musste der Datenverarbeiter ihn benachrichtigen. Neuartig, wie diese Benachrichtigungspflicht war, ließen sich ihre Folgen nicht absehen. Den Datenverarbeitern ging sie gegen den Strich; Sie befürchteten eine neue Kostenwelle, doch sie konnten sich nicht durchsetzen und mussten schließlich die Belastung hinnehmen.

Nach dem Inkrafttreten des Gesetzes stellte sich heraus, dass der Betroffene den Begriff "Schutz" als etwas verstand, das inm zu gewähren ist, nicht als etwas, um das er sich bemühen muss. Die Benachrichtigungspost beachtete er wenig; er verhielt sich passiv. Aber auch wenn er nicht im eigenen Interesse aktiv wurde, war er doch durch die gesetzlichen Auflagen an den Datenverarbeiter vor dessen Willkür geschützt. So geriet der Datenschutz einseitig zu einem Aufgabenbereich der Datenverarbeitung; strafbedroht setzten inn die Datenverarbeiter um. Sie setzten auch in der Folge die EU-Richtlinie eine zweite und eine dritte Welle von Datenschutzgesetzen und -verordnungen, Teledienstedatenschutzgesetz, Telemediengesetz etc um. Die Benachrichtigungspflicht wurde zur Unterrichtungspflicht ausgeweitet. Sie wurde von der Wirtschaft überraschend bereitwillig aufgegriffen. Derzeit wird bei praktisch jedem Vorfall der Kunde darüber unterrichtet, warum und wozu seine Daten benötigt werden. Wenn eine Übermittlung an Dritte vorgesehen ist, die das Gesetz im Zweifelsfalle nicht zuließe, wird der Betroffene nach Rechtsbelehrung um seine verbindliche Einwilligung gebeten. Dabei wird beteuert, dass die Daten des Betroffenen in guter Hand blieben. Diese Unterrichtung wird meist schon im Angebot deutlich herausgehoben und als "Datenschutzerklärung" bezeichnet.

So kann man der auferlegten Benachrichtigungs-/Unterrichtungspflichtpflicht nachkommen und kann, wenn erforderlich, per Einwilligung des Betroffenen der Datenschutz legal aushebeln. Die Wirtschaft, der in den 70er-Jahren die Benachrichtigungspflicht so sauer war, hat nun damit einen Mehrwert, einen neuartigen Werbeträger. Sie kann den Kunden mit ihrem guten Willen zum Datenschutz beeindrucken. So kommt sie mit Gewinn einer gesetzlichen Pflicht nach. Es ist aber, liebe Leserinnen und Leser, auch ein Gewinn für den Datenschutz. Wo sonst wird er so professionell propagiert?

Mit freundlichen Grüßen, Ihr

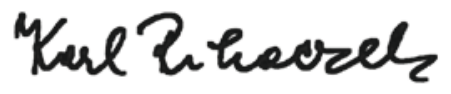

